\title{
Ökodesign von Stahlwerksschlacken durch thermochemische Behandlung zur Erhöhung der Recyclingfähigkeit
}

\author{
Daniel Vollprecht und Roland Pomberger
}

Lehrstuhl für Abfallverwertungstechnik und Abfallwirtschaft, Montanuniversität Leoben, Leoben, Österreich

Eingegangen 19. Januar 2021; angenommen 3. Februar 2021; online publiziert 24. Februar 2021

Zusammenfassung: Ökodesign ist ein Konzept zur Gestaltung von Produkten, dessen Ziel es ist, die Umweltbelastung zu verringern. Theoretische, praktische und reale Recyclingfähigkeit sind Eigenschaften eines Produktes, am Ende seines Lebenszyklus stofflich verwertet werden zu können, wobei "Recycling" stets impliziert, dass das Produkt zeitweilig zu Abfall wird.

Stahlwerksschlacken werden im pyrometallurgischen Prozess gezielt durch die Zugabe von Schlackenbildnern erzeugt und erfüllen dort eine Funktion, indem sie die im Stahl unerwünschten Begleitelemente der eingesetzten geogenen oder anthropogenen Erze aufnehmen. Stahlwerksschlacken sind somit jedenfalls im Hinblick auf die metallurgische Nutzungsphase Produkt, können aber an deren Ende - je nach Judikatur - Abfall werden oder Nebenprodukt sein. In beiden Fällen handelt es sich um Reststoffe, die als industriell hergestellte Gesteinskörnungen Sekundärrohstoffe für den Straßenbau darstellen.

Die thermochemische Behandlung von Stahlwerksschlacken im flüssigen Zustand kann die Freisetzung umweltrelevanter Elemente in Poren- und Grundwässer und damit die Umweltbelastung verringern, indem diese Elemente in stabilen Mineralphasen eingebaut und/oder nach einer anfänglichen Freisetzung wieder an deren Oberflächen adsorbiert werden können.

Diese gezielte Steuerung der Produktionsbedingungen von Stahlwerksschlacken stellt somit ein Beispiel für Ökodesign dar und ist zudem für jene Fälle, in denen die Schlacke zu Abfall wird, ein wesentlicher Beitrag zur Erhöhung der theoretischen Recyclingfähigkeit der Schlacke, die in diesem Spezialfall mit der praktischen und realen Recyclingfähigkeit zusammenfällt.

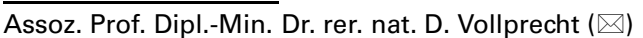
Lehrstuhl für Abfallverwertungstechnik und Abfallwirtschaft, Montanuniversität Leoben,

Franz-Josef-Str. 18,

8700 Leoben, Österreich

daniel.vollprecht@unileoben.ac.at
Schlüsselwörter: Ökodesign, Stahlwerksschlacke, Thermochemische Behandlung, Recyclingfähigkeit

EcoDesign of Steel Slags by Thermochemical Treatment for an Increase of Recyclability

Abstract: Ecodesign is a concept for the product development whose aim is to reduce environmental loads. Theoretical, practical, and real recyclability are properties of a product to be recycled at the end of its life cycle with "recycling" always implying that the product temporarily becomes a waste.

Steel slags are produced specifically in the pyrometallurgical process by the addition of slag formers and fulfil there a function by incorporating the accompanying elements undesired in the steel derived from the used geogenic or anthropogenic ores. Consequently, steel slags are-at least with respect to the metallurgical use phase-a product but can become waste or be a by-product at its end, depending on the judicature. In both cases slags are residues which, as industrially produced aggregates, represent secondary raw materials for road construction.

The thermochemical treatment of steel slags in the liquid state can decrease the release of environmentally relevant elements into pore and groundwaters and correspondingly the environmental impact by incorporation of these elements in stable mineral phases and/or after initial release adsorbed again at their surfaces.

This specific control of production conditions of steel slags consequently represents an example for EcoDesign and is therefore, for those cases in which slag becomes a waste, a significant contribution for the increase of the theoretical recyclability of the slag, which coincides in this specific case with the practical and real recyclability.

Keywords: EcoDesign, Steel slag, Thermochemical treatment, Recyclability 


\section{Einleitung}

Ökodesign ist ein Konzept zur Gestaltung von Produkten, dessen Ziel es ist, die Umweltbelastung zu verringern [1]. Meist wird der Begriff für Produkte, wie z. B. Batterien, verwendet [2]. Bezüglich industrieller Reststoffe wird bisher nur selten von Ökodesign gesprochen, z. B. im Hinblick auf die Synthese von Glaskeramiken aus Aufbereitungsrückständen und Papierreststoffverbrennungsaschen sowie von Adsorbenzien aus diesen Aschen, Kohleflugaschen und Stahlwerksschlacken [3]. Die Ursache dafür ist darin zu suchen, dass die Produkteigenschaft industrieller Reststoffe umstritten ist [4], wenn der Begriff "Produkt" in seiner abfallrechtlichen Bedeutung als Abgrenzung zu "Abfall“ und nicht in seiner verfahrenstechnischen Bedeutung als Abgrenzung zu "Edukt" verwendet wird.

Theoretische, praktische und reale Recyclingfähigkeit [5] sind Eigenschaften eines Produktes, am Ende seines Lebenszyklus stofflich verwertet werden zu können, wobei "Recycling" stets impliziert, dass das Produkt zeitweilig zu Abfall wird [6]. Recyclingfähigkeit ist eine Möglichkeit des Ökodesigns von Produkten, da das Recycling bzgl. vieler Wirkungskategorien gegenüber der Deponierung und dem Einsatz primärer Rohstoffe ökologisch vorteilhaft ist, wobei die Zuweisung der Umweltbelastungen industrieller Prozesse zu Produkten und Abfällen einen großen Einfluss hat [7]. Im Gegensatz zu anderen Möglichkeiten des Ökodesigns wird die Recyclingfähigkeit auch in Bezug auf industrielle Reststoffe oft untersucht, wobei im Hinblick auf metallurgische Schlacken unter "Recycling" unterschiedliche Prozesse verstanden werden. In einer Studie zur Recyclingfähigkeit von Schlacken der pyrometallurgischen Behandlung von Elektroaltgeräten [8] wurde darunter die Rückgewinnung von Metallen verstanden, wohingegen in einer Arbeit zur Recyclingfähigkeit von Mg-reichen Eisen-NickelSchlacken eine Verwertung als Gesteinskörnung gemeint ist [9]. Im Gegensatz zur Recyclingfähigkeit von Konsumgütern, wie z.B. PET-Flaschen, die von Faktoren wie dem Vorhandensein von Sammelsystemen ( $\rightarrow$ „reale Recyclingfähigkeit“) und der technischen Erkennbarkeit in mechanischen Abfallbehandlungsanlagen ( $\rightarrow$ „praktische Recyclingfähigkeit“) beeinflusst werden [5], ist die Recyclingfähigkeit industrieller Reststoffe fast ausschließlich durch die Materialeigenschaften bedingt, da sie bereits getrennt anfallen und nicht mehr von anderen Abfällen getrennt werden müssen. Die materialspezifische "theoretische Recyclingfähigkeit" [5] ist somit in diesem Spezialfall gleichbedeutend mit der „realen Recyclingfähigkeit."

Stahlwerksschlacken werden im pyrometallurgischen Prozess gezielt durch die Zugabe von Schlackenbildnern erzeugt und erfüllen dort eine Funktion, indem sie die im Stahl unerwünschten Begleitelemente der eingesetzten geogenen oder anthropogenen Erze aufnehmen [10]. Die metallurgische Nutzungsphase stellt somit den ersten Abschnitt des Lebenszyklus einer Schlacke dar. Während dieser Phase ist eine Schlacke nicht nur verfahrenstechnisch, sondern auch abfallrechtlich Produkt, so dass die Anwendung produktbezogener Begrifflichkeiten wie "Ökodesign" und "Recyclingfähigkeit" aus dieser Sicht gerechtfertigt ist. Am Ende dieser metallurgischen Nutzungsphase kön- nen Schlacken - je nach Judikatur - Abfall werden oder Nebenprodukt sein. Obwohl die Abgrenzung zwischen Abfall und Nebenprodukt anhand von vier Kriterien (sichere Weiterverwendung, direkte Verwendbarkeit, integraler Bestandteil eines Herstellungsprozesses, Rechtmäßigkeit der Verwendung) klar definiert ist [6], ist noch nicht hinreichend erforscht, warum in Österreich Stahlwerksschlacken aus dem Linz-Donawitz-Prozess als Abfall eingestuft werden, während Elektroofenschlacken der Marienhütte in Graz als Nebenprodukt anerkannt sind [11]. Diese Unterscheidung ist einerseits von akademischem Interesse, weil "Recycling" und damit auch „Recyclingfähigkeit" das Durchlaufen einer Lebensphase als Abfall voraussetzt, und andererseits von praktischer Bedeutung, da die Einstufung als Abfall entgegen der Abfallhierarchie, die das Recycling der Beseitigung vorzieht [6], die Verwendung des Materials oftmals hemmt, da beim Recycling von Abfällen strengere umweltrechtliche Anforderungen gelten, z. B. die Recyclingbaustoffverordnung [12], als bei der Verwendung von Nebenprodukten oder dem Einsatz von Produkten aus Primärrohstoffen. „Ökodesign“ umfasst hingegen im Gegensatz zur "Recyclingfähigkeit" auch jene Möglichkeiten, den Lebenszyklus eines Produkts zu verlängern, bei denen dieses niemals zu Abfall wird, wie z.B. die Wiederverwendung oder die Sicherstellung einer Einstufung als Nebenprodukt mit nachfolgender Verwendung. Somit ist der Begriff der "Recyclingfähigkeit" streng genommen nur für jene Schlacken anwendbar, die kein Nebenprodukt sind. Ein Ökodesign, das eine "Abfallwerdung" (z. B. durch Nebenproduktanerkennung) von vornherein verhindert, ist somit aufgrund der oben ausgeführten praktischen Auswirkung der Abfalleigenschaft der "Recyclingfähigkeit" eines Produkts vorzuziehen.

Unabhängig von der abfallrechtlichen Einstufung handelt es sich bei Stahlwerksschlacken um Reststoffe, die als industriell hergestellte Gesteinskörnungen Sekundärrohstoffe für den Straßenbau darstellen. Reststoffe sind Rückstände eines industriellen Prozesses, die sowohl Nebenprodukt als auch Abfall sein können [13]. Industriell hergestellte Gesteinskörnungen [12] sind somit Reststoffe, da sie nicht Hauptprodukt des jeweiligen industriellen Prozesses sind, aber auch Sekundärrohstoffe [14] für die Erzeugung hydraulisch oder bituminös gebundener Baustoffe, wie Beton bzw. Asphalt, wo sie natürlichen Gesteinskörnungen hinsichtlich ihrer mechanischen Eigenschaften oftmals überlegen sind [15], wobei natürlich immer die spezifische Schlacke und das spezifische Gestein verglichen werden müssen.

Da bergwirtschaftliche ( $R$ Ressource“) , verfahrenstechnische ( „Reststoff“) und abfallwirtschaftliche („Abfall“) Begriffe oftmals nicht miteinander in Beziehung gesetzt werden, soll dies hier geschehen und die Schlacken in diese Begrifflichkeiten eingeordnet werden (Abb. 1).

\section{Thermochemische Behandlung von Stahlwerksschlacken}

Die baustoffliche Recyclingfähigkeit einer als Abfall eingestuften Schlacke bzw. die Zulässigkeit der Verwendung ei- 
Abb. 1: Einordnung von Schlacken in bergwirtschaftliche, verfahrenstechnische und abfallwirtschaftliche Begrifflichkeiten

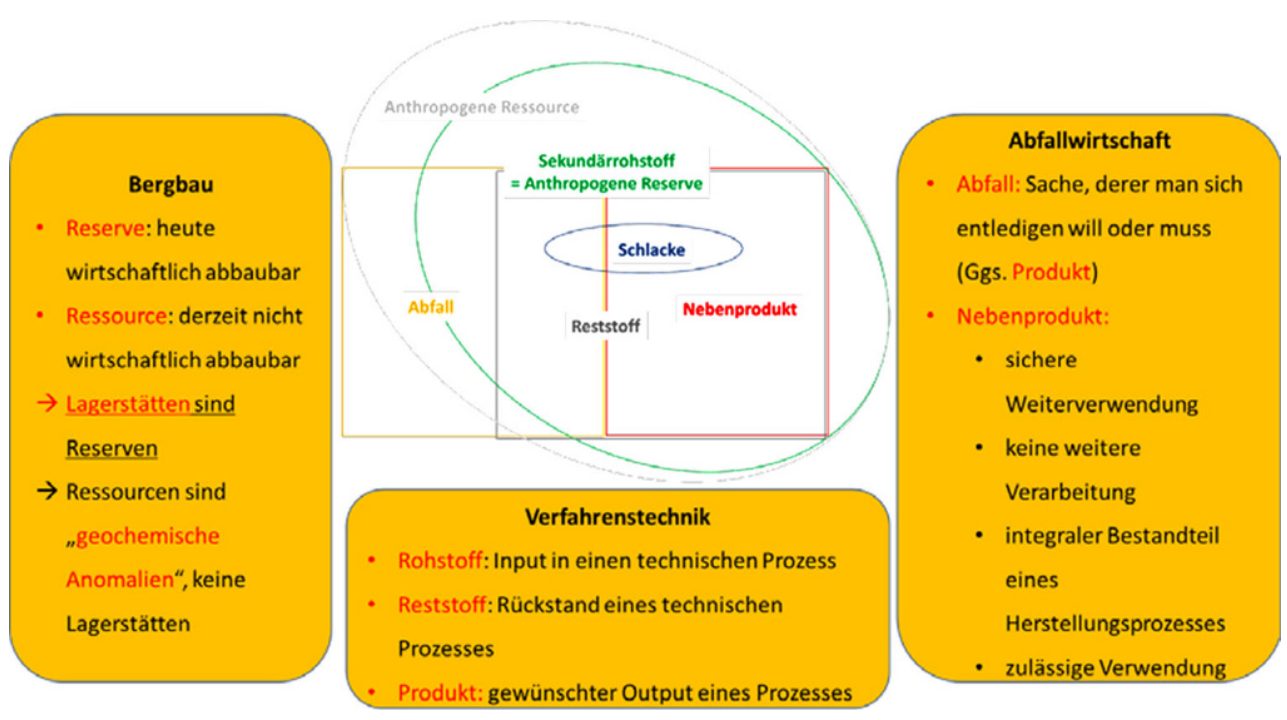

ner Schlacke als Baustoff als Voraussetzung für eine Einstufung als Nebenprodukt hängen von der Freisetzung umweltrelevanter Elemente (z. B. Cr, V, Mo, F) in die Umwelt ab. Sowohl natürliche als auch industriell erzeugte Gesteinskörnungen können derartige Schadstoffe in die Umwelt freisetzen [16], dennoch werden sie in Österreich mit zweierlei Maß gemessen, da entsprechende Grenzwerte für Eluatkonzentrationen nur für industriell erzeugte und rezyklierte, nicht aber für natürliche Gesteinskörnungen existieren. Bei der Beurteilung der Zulässigkeit der Verwendung eines Nebenprodukts gelten die Grenzwerte der Recyclingbaustoffverordnung zwar nicht, werden aber mittelbar in die Bewertung mit einbezogen. Erschwerend kommt hinzu, dass die Recyclingfähigkeit industrieller Gesteinskörnungen nicht nur anhand von Eluatkonzentration, sondern in Österreich - im Gegensatz z. B. zu Deutschland und den Niederlanden - auch durch Gesamtgehalte (in Deutschland als Feststoffgehalte bezeichnet) an umweltrelevanten Elementen beschränkt wird. Dies steht im eklatanten Widerspruch zum Stand der Wissenschaft, demzufolge die Auslaugbarkeit chemischer Elemente nicht von deren Gesamtgehalten, sondern von ihrer mineralogischen Bindung abhängt [17].

Industriell erzeugte Gesteinskörnungen haben gegenüber natürlichen Gesteinskörnungen einen wesentlichen umwelttechnischen Vorteil: Während die mineralogische Bindungsform umweltrelevanter Elemente in natürlichen Gesteinen über geologische Zeiträume hinweg entstanden ist und durch den Menschen nicht mehr verändert werden kann, kann die Mineralogie industriell erzeugter Gesteinskörnungen wie Stahlwerksschlacken gezielt im pyrometallurgischen Prozess eingestellt werden, indem Prozessparameter wie der $\mathrm{MgO}-\mathrm{Gehalt}$ oder das $\mathrm{CaO} / \mathrm{SiO}_{2}-$ Verhältnis im Hinblick auf eine Minimierung der Auslaugbarkeit umweltrelevanter Elemente wie z. B. Chrom optimiert werden [18]. Die Zugabe chemischer Elemente und Verbindungen zu einem Hochtemperaturprozess mit dem Ziel, dort gewisse chemische Reaktionen, wie z. B. die Bildung bestimmter Mineralphasen, zu bewirken, wird als thermochemische Behandlung bezeichnet, stellt eine wesentliche Möglichkeit zur Abfallvermeidung in der Stahlindustrie [19] bzw. zur Erhöhung der Recyclingfähigkeit von Stahlwerksschlacken dar und ist somit eine Schlüsseltechnologie zum Ökodesign industrieller Reststoffe.

Ungefähr seit der Jahrtausendwende wurden umfangreiche Forschungsarbeiten zur thermochemischen Behandlung von Stahlwerksschlacken durchgeführt, die auf einer grundlegenden Ebene begannen [18] und inzwischen für LD-Schlacken durch die thyssenkrupp Steel Europe AG in Form des Lidonit ${ }^{\circledR}$ industriell umgesetzt wurde [20].

Zunächst gelang es am FEhS-Institut für Baustoffforschung mit dem Faktor sp zu aufgrund des Verhältnisses vom spinell- und silikatgebundem Chrom für Edelstahlschlacken die Auslaugbarkeit von Chrom vorherzusagen [21]. Später wurde an synthetischen Proben gezeigt, dass mit abnehmendem $\mathrm{CaO} / \mathrm{SiO}_{2}$-Verhältnis die Bildung $\mathrm{Cr}(\mathrm{V})$ und $\mathrm{Cr}(\mathrm{VI})$-haltiger Mineralphasen wie der Apatit-artigen Verbindung [22] $\mathrm{Ca}_{5}\left(\mathrm{CrO}_{4}\right)_{3} \mathrm{~F}$ bzw. dem Chromatit, $\mathrm{CaCrO}_{4}$, unterbunden werden kann und die Zugabe von $\mathrm{MgO}$ die Bildung von Spinellen und die Einbindung von $\mathrm{Cr}$ (III) in diese Mineralgruppe fördert [18]. Zudem konnte gezeigt werden, dass der Einbau von Chrom in Spinelle, $(\mathrm{Fe}, \mathrm{Mg})(\mathrm{Al}, \mathrm{Cr})_{2} \mathrm{O}_{4}$, auch in Elektroofenschlacken aus der Baustahlerzeugung die Ursache für die äußerst geringe Auslaugung von Chrom ist [17].

Nachdem klar wurde, dass die Freisetzung von Chrom aus Stahlwerksschlacken in der Regel kein Problem darstellt und andernfalls durch entsprechende Konditionierungsmaßnahmen leicht in den Griff zu bekommen ist, verschob sich der Fokus auf das Vanadium. Aufbauend auf der Beobachtung, dass die Auslaugbarkeit von Vanadium aus LD-Schlacken im Vergleich zum Chrom trotz deutlich geringerer Gesamtgehalte höher ist, konnte mittels mineralogischer Untersuchungen die Erklärung gefunden werden: Obwohl beide Elemente laut Elektronenmikroskopie in derselben Phase, Dicalciumferrit, $\mathrm{Ca}_{2} \mathrm{FeO}_{4}$, gebunden sind, zeigte die Röntgennahkantenabsorptionsspektroskopie (XANES), dass Chrom stabil als $\mathrm{Cr}$ (III) vorliegt und bei Alterung und Auslaugung auch nicht oxidiert wird, wäh- 


\begin{tabular}{|c|c|c|}
\hline Publikation & Ergebnis & Bedeutung für die Abfallwirtschaft \\
\hline $\begin{array}{l}\text { Cheremisina \& } \\
\text { Schenk (2017) [30] }\end{array}$ & $\begin{array}{l}\text { Keine Entstehung von } \mathrm{Cr}(\mathrm{VI}) \text { bei der Erstarrung von } \\
\text { Stahlwerksschlacken }\end{array}$ & $\begin{array}{l}\text { Umweltrisiko der Auslaugung von } \mathrm{Cr}(\mathrm{VI}) \\
\text { gering }\end{array}$ \\
\hline $\begin{array}{l}\text { Höllen et al. (2016) } \\
\text { [31] }\end{array}$ & $\begin{array}{l}\text { Starke Schwankungen der Gesamtgehalte und Eluat- } \\
\text { konzentrationen }\end{array}$ & $\begin{array}{l}\text { Identifizierung oder Vermeidung von Fehl- } \\
\text { chargen }\end{array}$ \\
\hline \begin{tabular}{|l|} 
Höllen et al. (2017) \\
[32] \\
\end{tabular} & Konzept des lokalen Gleichgewichts & $\begin{array}{l}\text { Erklärung des Elutionsverhaltens nur durch } \\
\text { aufwändige Verfahrenskombinationen }\end{array}$ \\
\hline $\begin{array}{l}\text { Neuhold et al. } \\
\text { (2018a) [33] }\end{array}$ & $\begin{array}{l}\text { Stabiler Einbau von } \mathrm{Cr} \text { und } \mathrm{V} \text { in Spinelle und Adsorp- } \\
\text { tion von } \mathrm{V}\end{array}$ & $\begin{array}{l}\text { Geringe Freisetzung von } \mathrm{Cr} \text { und } \mathrm{V} \text { aus Elek- } \\
\text { troofenschlacken }\end{array}$ \\
\hline $\begin{array}{l}\text { Neuhold et al. } \\
\text { (2018b) [34] }\end{array}$ & $\mathrm{Cr}(\mathrm{III})$ und $\mathrm{V}$ (III) werden im Festkörper nicht oxidiert & $\begin{array}{l}\text { Keine Hinweise auf Gefahren durch Oxidation } \\
\text { von } \mathrm{Cr} \text { und } \mathrm{V}\end{array}$ \\
\hline $\begin{array}{l}\text { Neuhold et al. } \\
\text { (2018c) [35] }\end{array}$ & \multirow[t]{2}{*}{$\begin{array}{l}\text { Sowohl natürliche als auch industrielle } \\
\text { Gesteinskörnungen können Grenzwerte überschreiten }\end{array}$} & \multirow{2}{*}{$\begin{array}{l}\text { Keine Rechtfertigung für Ungleichbehandlung } \\
\text { natürlicher und industrieller } \\
\text { Gesteinskörnungen hinsichtlich } \\
\text { umwelttechnischer Anforderungen }\end{array}$} \\
\hline $\begin{array}{l}\text { Vollprecht et al. } \\
\text { (2019) [36] }\end{array}$ & & \\
\hline $\begin{array}{l}\text { Neuhold et al. } \\
\text { (2019b) [37] }\end{array}$ & $\begin{array}{l}\text { Primäre partielle Freisetzung und sekundäre Fixierung } \\
\text { von Vanadium }\end{array}$ & $\begin{array}{l}\text { Gezielte Immobilisierung von Vanadium ist } \\
\text { möglich }\end{array}$ \\
\hline $\begin{array}{l}\text { Neuhold et al. } \\
\text { (2018d) [38] }\end{array}$ & \multirow[t]{2}{*}{$\begin{array}{l}\text { Verringerung der V-Auslaugung durch } \mathrm{SiO}_{2} \text {-Zugabe } \\
\text { zur flüssigen Schlacke im Labormaßstab }\end{array}$} & \multirow{3}{*}{$\begin{array}{l}\text { Optimierung der Recyclingfähigkeit von } \\
\text { Schlacken durch thermochemische } \\
\text { Konditionierung möglich }\end{array}$} \\
\hline $\begin{array}{l}\text { Neuhold et al. } \\
\text { (2019a) [39] }\end{array}$ & & \\
\hline $\begin{array}{l}\text { Neuhold et al. (2020) } \\
\text { [40] }\end{array}$ & $\begin{array}{l}\text { Verringerung der V-Auslaugung durch } \mathrm{SiO}_{2} \text {-Zugabe } \\
\text { zur flüssigen Schlacke im Technikums-maßstab }\end{array}$ & \\
\hline
\end{tabular}

rend Vanadium als V(IV) vorliegt und zu V(VI) oxidiert wird, was sowohl eine höhere Mobilität als auch eine höhere Toxizität bewirkt $[23,24]$. In anderen LD-Schlacken blieb Vanadium im Dicalciumferrit hingegen stabil, war aber auch im Dicalciumsilicat, $\mathrm{Ca}_{2} \mathrm{SiO}_{4}$, gebunden und wurde aus diesem herausgelöst, dann aber teilweise wieder in Calcium-Silikat-Hydratphasen gebunden [25]. Die Beobachtung, dass Vanadium aus Silikatphasen mit einem geringen Polymerisationsgrad der $\mathrm{SiO}_{4}$-Tetraeder, wie z. B. dem Inselsilikat $\mathrm{Ca}_{2} \mathrm{SiO}_{4}$, relativ leicht gelöst werden kann [26], legt die Grundlage für das auch von ThyssenKrupp genutzte thermochemische Konditionierungsprinzip der $\mathrm{SiO}_{2}$-Zugabe zur flüssigen Schlacke. Hierdurch bilden sich eher Gruppensilikate wie Gehlenit, $\mathrm{Ca}_{2} \mathrm{Al}^{[4]}\left[\mathrm{AISiO}_{7}\right]$, deren Löslichkeit und hydraulisches Verhalten geringer sind. Dass dieses Verfahren nicht nur für LD-Schlacken, sondern auch für Elektroofenschlacken funktioniert, konnte bereits gezeigt werden [27].

Obwohl die thermochemische Behandlung von Stahlwerksschlacken somit in vielen Fällen erfolgreich angewendet werden konnte, gibt es auch gegenteilige Beobachtungen: Für eine Pfannenschlacke wurde beispielsweise gezeigt, dass eine Modifizierung der chemischen Zusammensetzung zwar die mineralogische Zusammensetzung, nicht aber die Auslaugbarkeit verändert [28].

\section{Das Projekt MiLeSlag}

Um das Verständnis über den Zusammenhang zwischen der Mineralogie und dem Auslaugverhalten von Stahlwerksschlacken zu vertiefen und Grundlagen für eine Optimierung des Auslaugverhaltens zu legen, wurde zwischen 2016 und 2019 das Forschungsprojekt „MiLeSlag“ (FFG,
Bridge Frühphase, Projekt-Nummer 851210) von einem internationalen Konsortium, bestehend aus der Montanuniversität Leoben, der Bundesanstalt für Materialforschung und -prüfung, dem Energy Research Centre of the $\mathrm{Ne}$ therlands, dem FEhS-Institut für Baustoffforschung, der Marienhütte Stahl- und Walzwerk $\mathrm{GmbH}$, der Max Aicher Umwelt $\mathrm{GmbH}$, der Porr Umwelttechnik $\mathrm{GmbH}$ und der Scholz Austria $\mathrm{GmbH}$, durchgeführt. Im Projekt „MiLeSlag" wurden vier peer-reviewte und sieben weitere Artikel veröffentlicht, in denen die wichtigsten Ergebnisse des Projekts zusammengefasst wurden. Die abfallwirtschaftliche Relevanz der erzielten Projektergebnisse wurde bereits in einem Beitrag zur Recy\&DepoTech 2020 dargestellt [29], da sie zuvor von der österreichischen Abfallwirtschaft nicht hinreichend wahrgenommen worden wurde. Daher soll an dieser Stelle der Überblick aus diesem Tagungsbandbeitrag direkt wiedergegeben werden [29]:

Cheremisina \& Schenk (2017) [30] zeigten anhand thermodynamischer Modellierungen, dass sich während des pyrometallurgischen Prozesses kein sechswertiges Chrom in Stahlwerksschlacken bilden kann. Für die Abfallwirtschaft bedeutet dies, dass die Diskussion über die Umweltauswirkungen von Stahlwerksschlacken bzgl. Chrom weitgehend geklärt ist, da dieses - wenn überhaupt - nur in Spuren in späteren Verwitterungsprozessen entstehen könnte.

Höllen et al. (2016) [31] stellten dar, dass die Gesamtgehalte und Eluatkonzentrationen von Chrom, Vanadium und Molybdän zwischen verschiedenen Produktionschargen desselben Stahlwerks um rund eine Zehnerpotenz schwanken, wobei insbesondere die Eluatkonzentrationen rechtsschiefe Verteilungen aufweisen. Für die Abfallwirtschaft bedeutet dies, dass Wege gefunden werden müssen, die wenigen Chargen, deren Schwermetallkonzentra- 
tionen über etwaigen Grenzwerten liegen, zu erkennen und abzutrennen bzw. langfristig ihre Produktion durch Einstellung geeigneter Prozessbedingungen zu vermeiden.

Höllen et al. (2017) [32] entwickelten ein Modell, demzufolge sich die Oberfläche zwischen Stahlwerkschlacke und wässriger Lösung in einem lokalen Gleichgewicht befindet, die Minerale im Inneren der Schlacke jedoch nur miteinander und nicht mit der Oberfläche und der wässrigen Lösung im Gleichgewicht stehen. Das Bindeglied, das die Hochtemperaturprozesse im Stah/werk und damit die Mineralogie des Inneren der Schlacke mit den Niedertemperaturprozessen in der Umwelt und damit der Oberfläche der Schlacke verknüpft, fehlt hingegen noch. Somit liefern weder hydrogeochemische Modellierungen noch mineralogische Untersuchungen allein, sondern nur eine Kombination beider Ansätze eine Erklärung für das Auslaugverhalten von Stahlwerksschlacken. Derartig aufwändige Verfahrenskombinationen sind in der Abfallwirtschaft selten, liefern aber argumentative Hilfestellungen zum Nachweis der zulässigen Verwertung oder der Nebenprodukteigenschaft von Stah/werksschlacken. Neuhold et al. (2018a) identifizierten die stabile Bindung von dreiwertigem $\mathrm{Cr}$ und $\mathrm{V}$ in Spinellmischkristallen, und die Adsorption von $V$ an sekundären Mineralphasen als möglichen auslaugbestimmenden Mechanismus. Für die Abfallwirtschaft bedeutet dies, dass $\mathrm{Cr}$ und $\mathrm{V}$ in Stahlwerksschlacken unter gewissen Prozessbedingungen stabil gebunden sind oder durch gezielte Einstellung der Mineralogie stabil gebunden werden könnten.

Neuhold et al. (2018b) [33] wandten erstmals die Röntgennahkantenabsorptionsspektrokopie (XANES) zur ortsaufgelösten Bestimmung der Wertigkeit von $\mathrm{Cr}$ und $\mathrm{V}$ in EOS an und zeigten, dass dies dreiwertig vorliegt und sich bei der Auslaugung nicht verändert. Dies bedeutet für die Abfallwirtschaft, dass Ergebnisse zur dreiwertigen Oxidationszahl von Chrom bei hohen Temperaturen (Cheremisina et al. 2017) auf das Verhalten beim Einbau von EOS als Recyclingbaustoff übertragen werden können.

Neuhold et al. (2018c) [34] und Vollprecht et al. (2019) [35] zeigten, dass sowohl industrielle Gesteinskörnungen (EOS) als auch natürliche Gesteinskörnungen teilweise Grenzwerte bzgl. der Eluatkonzentrationen oder Gesamtgehalte potenziell umweltrelevanter Elemente überschreiten können. Aus abfallwirtschaftlicher Sicht ist daher die Ungleichbehandlung industrieller und natürlicher Gesteinskörnungen hinsichtlich umwelttechnischer Anforderungen nicht zu rechtfertigen.

Neuhold et al. (2019b) [36] stellten fest, dass $V$ zunächst in Spinellen und Wüstit sowie in geringerem Ausmaß in Olivin gebunden ist, aus letzterem gelöst werden kann, dann aber wiederum an Calciumsilikathydratphasen fixiert wird. Diese Erkenntnis liefert weitere Grundlagen für die thermochemische Konditionierung von Elektroofenschlacken zur Optimierung ihrer Recyclingfähigkeit.

Neuhold et al. (2018d) [37] und Neuhold et al. (2019a) [38] zeigten in Laborversuchen, dass die Zugabe von $\mathrm{SiO}_{2}$ und damit die Verringerung des $\mathrm{FeO} / \mathrm{SiO}_{2}$-Verhältnisses in der flüssigen Schlacke die Auslaugung von Vanadium signifikant verringert. Für die Abfallwirtschaft bedeutet dies, dass
Schlacken gezielt so erzeugt werden können, dass ihre Recyclingfähigkeit verbessert werden kann.

Neuhold et al. (2020) gelang schließlich das Upscaling der Konditionierung in den Technikumsmaßstab mit einer Bestätigung des Effekts einer Verringerung der Auslaugung von $\mathrm{Cr}$ und $\mathrm{V}$ durch die gezielte Änderung der chemischen Zusammensetzung der flüssigen Schlacke.

Eine Zusammenfassung der abfallwirtschaftlichen Bedeutung der einzelnen Ergebnisse des Projekts liefert Tab. 1.

[... ]. Die Ergebnisse des Projekts "MiLeSlag“ zeigen, dass - Gesamtgehalte kein geeignetes Kriterium zur Umweltbewertung von Stahlwerksschlacken sind,

- die Ungleichbehandlung natürlicher und industrieller Gesteinskörnungen nicht gerechtfertigt ist und

- die Recyclingfähigkeit industrieller Gesteinskörnungen durch thermochemische Konditionierung optimiert werden kann [29].

\section{Zusammenfassung}

Die thermochemische Behandlung von Stahlwerksschlacken im flüssigen Zustand kann die Freisetzung umweltrelevanter Elemente in Poren- und Grundwässer und damit die Umweltbelastung verringern, indem diese Elemente in stabilen Mineralphasen eingebaut und/oder nach einer anfänglichen Freisetzung wieder an deren Oberflächen adsorbiert werden können.

Diese gezielte Steuerung der Produktionsbedingungen von Stahlwerksschlacken stellt somit ein Beispiel für Ökodesign dar und ist zudem für jene Fälle, in denen die Schlacke zu Abfall wird, ein wesentlicher Beitrag zur Erhöhung der theoretischen Recyclingfähigkeit der Schlacke, die in diesem Spezialfall mit der praktischen und realen Recyclingfähigkeit zusammenfällt.

Funding. Open access funding provided by Montanuniversität Leoben.

Open Access Dieser Artikel wird unter der Creative Commons Namensnennung 4.0 International Lizenz veröffentlicht, welche die Nutzung, Vervielfältigung, Bearbeitung, Verbreitung und Wiedergabe in jeglichem Medium und Format erlaubt, sofern Sie den/die ursprünglichen Autor(en) und die Quelle ordnungsgemäß nennen, einen Link zur Creative Commons Lizenz beifügen und angeben, ob Änderungen vorgenommen wurden.

Die in diesem Artikel enthaltenen Bilder und sonstiges Drittmaterial unterliegen ebenfalls der genannten Creative Commons Lizenz, sofern sich aus der Abbildungslegende nichts anderes ergibt. Sofern das betreffende Material nicht unter der genannten Creative Commons Lizenz steht und die betreffende Handlung nicht nach gesetzlichen Vorschriften erlaubt ist, ist für die oben aufgeführten Weiterverwendungen des Materials die Einwilligung des jeweiligen Rechteinhabers einzuholen.

Weitere Details zur Lizenz entnehmen Sie bitte der Lizenzinformation auf http://creativecommons.org/licenses/by/4.0/deed.de.

\section{Literatur}

1. Karlsson, R.; Luttropp, C.: EcoDesign: what's happening? An overview of the subject area of EcoDesign and of the papers in this special issue, Journal of Cleaner Production, 14 (2006), pp 1291-1298 
2. Schwarz, T.; Schopf, K.; Arnberger, A.: Reducing Conflicts of Interests in Eco-design: The Relation of Innovation Management and Eco-design in the Automotive Sector, in Matsumoto M., Masui K., Fukushige S., Kondoh S. (eds): Sustainability Through Innovation in Product Life Cycle Design. EcoProduction (Environmental Issues in Logistics and Manufacturing). Springer, Singapore, 2017, pp 33-45

3. Okada, K.; Kameshima, Y.; Nakajima, A.: Eco-Design of $\mathrm{CaO}-\mathrm{Al}_{2} \mathrm{O}_{3}$ ( $\left.\mathrm{Fe}_{2} \mathrm{O}_{3}\right)-\mathrm{SiO}_{2}$ System, in: Proceeding of International Symposium on EcoTopia Science, 2007, pp 745-749

4. Moser-Marzi, E.; Frühwirth, W.: Die Abgrenzung von Nebenprodukt und Abfall in der Industrie, in: Memorandum - Abfallwirtschaft, 2015, p 1-36, https://www.moser-marzi.at/wp-content/uploads/ Studie_Nebenprodukt-Abfall_2015.pdf. Zugegriffen: 15 Jan 2021

5. Pomberger, R.: Über theoretische, praktische und reale Recyclingfähigkeit, Österreichische Wasser- und Abfallwirtschaft, 72 (2020), S. $19-20$

6. Richtlinie 2008/98/EG des Europäischen Parlaments und des Rates vom 19. November 2008 über Abfälle und zur Aufhebung bestimmter Richtlinien

7. Chen, C.; Habert, G.; Bouzidi, Y.; Jullien, A.; Ventura, A.: LCA allocation procedure used as an incitative method for waste recycling: An application to mineral additives in concrete, Resources, Conservation and Recycling, 54 (2010), no 12, pp 1231-1240

8. Buchmann, M.; Borowski, N.; Leißner, T.; Heinig, T.; Reuter, M. Friedrich, B.; Peuker, U.: Evaluation of Recyclability of a WEEE Slag by Means of Integrative X-Ray Computer Tomography and SEMBased Image Analyses, Minerals, 10 (2020), no 4, p 309

9. Zhai, M.; Zhu, H.; Liang, G.; Wu, Q.; Zhang, C.; Hua, S.; Zhang, Z.: Enhancing the recyclability of air-cooled high-magnesium ferronickel slag in cement-based materials: A study of assessing soundness through modifying method, Construction and Building Materials, 261 (2020), p 120523

10. Matsuura, H.; Manning, C.; Fortes, R.; Fruehan, R.: Development of a Decarburization and Slag Formation Model for the Electric Arc Furnace, ISIJ International, 48 (2008), no 9, pp 1197-1205

11. Landesverwaltungsgericht Steiermark: LVwG 46.23-141/2018-, 2018

12. Bundesminister für Land- und Forstwirtschaft, Umwelt und Wasserwirtschaft: Recycling-Baustoffverordnung-RBV. BGBI. II Nr. 181/2015, 2015

13. Vollprecht, D.: Exploration, Mobilization and Fixation of Constituents of Mineral Wastes, Landfills, Contaminated Sites and Waste Waters, Habilitation, Lehrstuhl für Abfallverwertungstechnik und Abfallwirtschaft, Leoben: Montanuniversität, 2020

14. Prochorow, A. (Ed.): Secondary raw material, in: The Great Soviet Encyclopedia, 1970-1979, https://encyclopedia2.thefreedictionary. com/Secondary+Raw+Material (01.04.2020)

15. Faleschini, F.; Fernández-Ruíz, M.A.; Zanini, M.A.; Brunelli, K.; Pellegrino, C.; Hernández-Montes, E.: High performance concrete with electric arc furnace slag as aggregate: Mechanical and durability properties, Construction and Building Materials, 101 (2015), no 1, pp 113-121

16. Tossavainen, M.; Forssberg, E.: The potential leachability from natural road construction materials, Science of the Total Environment, 239 (1999), pp 31-47

17. Aldrian, A.; Raith, J.G.; Höllen, D.; Pomberger, R.: Influence of Chromium Containing Spinels in an Electric Arc Furnace Slag on the Leaching Behaviour, Journal of Solid Waste Technology and Management, 41 (2015), pp 357-365

18. Cabrera-Real, H.; Romero-Serrano, A.; Zeifert, B.; Hernandez-Ramirez, A.; Hallen-Lopez, M.; Cruz-Ramirez, A.: Effect of $\mathrm{MgO}$ and $\mathrm{CaO} / \mathrm{SiO}_{2}$ on the immobilization of chromium in synthetic slags, Journal of Material Cycles and Waste Management, 14 (2012), pp 317-324

19. Adam, C.; Schraut, K.; Weingart, E.; Hamann, C: Technische Möglichkeiten der Abfallvermeidung in der Stahlproduktion, in Recy\&DepoTech, Leoben, 2020, $9 \mathrm{~S}$.

20. thyssenkrupp Steel Europe AG, https://www.thyssenkrupp-mss. com/media/gabriele/unternehmen/publikationen/datenblatt_lidonit. pdf Zugegriffen: 17 Jan 2021

21. Mudersbach, D.: Verbesserung der Eigenschaften von Elektroofenschlacken aus der Herstellung von nichtrostenden Stählen zur Nutzung dieser Schlacken im Verkehrsbau, in: Schriftenreihe des FEhS-
Instituts für Baustoff-Forschung e.V, FEhS-Institut für Baustoff-Forschung, Band 11, Duisburg, 2004, $83 \mathrm{~S}$.

22. Bazuev, G.; Tyutyunnik, A.; Kuznetsov, M.; Samigullina, R.: Apatitelike Complex Oxides in the $\mathrm{Ca}-\mathrm{Cr}-\mathrm{Cu}-\mathrm{O}$ system: Synthesis, Crysta Structure, XPS and Magnetic Study, European Journal of Inorganic Chemistry, 34 (2016), pp 5340-5346

23. Chaurand, P.; Rose, J.; Domas, J.; Bottero, J.Y.: Speciation of $\mathrm{Cr}$ and $\mathrm{V}$ within BOF steel slag reused in road constructions, Journal of Geochemical Exploration, 88 (2006), pp 10-14

24. Chaurand, P.; Rose, J.; Briois, V.; Olivi, L.; Hazemann, J.L.; Proux, O.; Domas, J.; Bottero, J.Y.: Environmental impacts of steel slag reused in road construction: A crystallographic and molecular (XANES) approach, Journal of Hazardous Materials, 139 (2007), pp 537-542

25. Hobson, A. J.; Stewart, D. I.; Bray, A. W.; Mortimer, R. J. G.; Mayes, W. M.; Rogerson, M.; Burke, I.T.: Mechanism of Vanadium Leaching during Surface Weathering of Basic Oxygen Furnace Steel Slag Blocks: A Microfocus X-ray Absorption Spectroscopy and Electron Microscopy Study, Environmental Science and Technology, 51 (2017), pp 7823-7830

26. Van Zomeren, A.; van der Laan, S. R.; Kobesen, H. B. A.; Huijgen W. J. J.; Comans, R. N. J. Changes in mineralogical and leaching properties of converter steel slag resulting from accelerated carbonation at low $\mathrm{CO}_{2}$ pressure, Waste Management, 31 (2011), pp 2236-2244

27. Mombelli, D.; Mapelli, C.; Barella, S.; Gruttadauria, A.; Saout, G. Le Garcia-diaz, E. The efficiency of quartz addition on electric arc furnace (EAF) carbon steel slag stability, Journal of Hazardous Materials, 279 (2014), pp 586-596

28. Loncnar, M.; van der Sloot, H. A.; Mladenovič, A.; Zupančič, M.; Kobal, L.; Bukovec, P. Study of the leaching behaviour of ladle slags by means of leaching tests combined with geochemical modelling and mineralogical investigations. Journal of Hazardous Materials, 317 (2016), pp 147-157

29. Vollprecht, D., Neuhold, S. F. \& Pomberger, R.: Was bedeuten neue Erkenntnisse zur Schlackenkonditionierung für die Abfallwirtschaft? In Tagungsband zur 15. Recy\&DepoTech (2020), S. 619-622

30. Cheremisina, E.; Schenk, J.: Chromium Stability in Steel Slags. Steel Research International, 88 (2017), no 11, p 1700206

31. Höllen, D.; Neuhold, S.; Mudersbach, D.; Schüler, S.; Sommerauer, H.; Griessacher, T.; Dijkstra, J.; van Zomeren, A.: MiLeSlag Zusammenhang zwischen Mineralogie und Auslaugbarkeit von Stahlwerksschlacken, in Schlacken-Symposium, Meitingen, 2016, S. 125-139

32. Höllen, D.; Neuhold, S.; Mudersbach, D.; Schüler, S.; Sommerauer H.; Griessacher, T.; Dijkstra, J.; van Zomeren, A.; Presoly, P.; Schenk, J.; Pomberger, R.: Möglichkeiten und Grenzen von Modellen zur Vorhersage der Auslaugbarkeit von Stahlwerksschlacken, in Mineralische Nebenprodukte und Abfälle 4, Berlin, 2017, S. 205-222

33. Neuhold, S.; Höllen, D.; van Zomeren, A.; Dijkstra, J.: Welche Mechanismen kontrollieren die Auslaugung von Schwermetallen aus Stahlwerksschlacken? - Verknüpfung von Experiment und Modell, in: Wissenschaftskongress Abfall- und Ressourcenwirtschaft 8 , Wien, 2018, S. 47-51

34. Neuhold, S.; Höllen, D.; Pomberger, R.; Mudersbach, D.; Schüler, S.; van Zomeren, A.: Einfluss der Auslaugung auf die Oberfläche von Stahlwerksschlacken, in Mineralische Nebenprodukte und Abfälle 5, Berlin, 2018b, S. 251-263

35. Neuhold, S.; Vollprecht, D.; Raith, J.G.; Berger, M.; Schüler, S.; Mudersbach, D.: Auslaugverhalten von Stahlwerksschlacken und natürlichen Gesteinskörnungen - ein Vergleich, in Schlacken-Symposium, Meitingen, 2018c, S. 179-189

36. Vollprecht, D.; Berger, M.; Altenburger-Junker, I.; Neuhold, S.; Sedlazeck, K.P.; Aldrian, A.; Dijkstra, J.J.; van Zomeren, A.; Raith J.G.: Mineralogy and Leachability of Natural Rocks-A Comparison to Electric Arc Furnace Slags, Minerals 9 (2019), no 8, pp 501

37. Neuhold, S.; van Zomeren, A.; Dijkstra, J.J.; van der Sloot, H.A.; Drissen, P.; Algermissen, D.; Mudersbach, D.; Schüler, S.; Griessacher, T.; Raith, J.G.; Pomberger, R.; Vollprecht, D.: Identification of Leaching Controlling Mechanisms for Chromium and Vanadium in Electric Arc Furnace (EAF) Slags Using New Experimental and Combined Modelling Approaches, Minerals, 9 (2019b), no 9, p 525 
38. Neuhold, S.; Vollprecht, D.; Presoly, P.; Schenk, J.; Adamczyk, B.: Freisetzungsbestimmende Mechanismen von Schwermetallen in Stahlwerksschlacken - Analyse, Modellierung, Synthesen, in Recy \& DepoTech 2018d: Vorträge-Konferenzband, Leoben, S. 135-140

39. Neuhold, S.; Vollprecht, D.; Presoly, P.; Adamczyk, B.; Drissen, P.; van Zomeren, A.; Schüler, S.; Mudersbach, D.: Minimising the leaching of $\mathrm{V}$ and $\mathrm{Cr}$ from electric arc furnace slags by decreasing the $\mathrm{FeO} / \mathrm{SiO}_{2}$ ratio, in 6th International Slag Valorisation Symposium, Mechelen, 2019a, pp 175-178

40. Neuhold, S.; Algermissen, D.; Drissen, P.; Adamczyk, B.; Presoly, P.; Sedlazeck, K. P.; Schenk, J.; Raith, J. G.; Pomberger, R.; Vollprecht, D.: Tailoring the $\mathrm{FeO} / \mathrm{SiO}_{2}$ Ratio in Electric Arc Furnace Slags to Minimize the Leaching of Vanadium and Chromium. Applied Sciences 10 (2020), no 7, p 2549

Hinweis des Verlags. Der Verlag bleibt in Hinblick auf geografische Zuordnungen und Gebietsbezeichnungen in veröffentlichten Karten und Institutsadressen neutral. 